\title{
Progranulin Protects Against Airway Remodeling Through the Modulation of Autophagy via HMGBI Suppression in House Dust Mite-Induced Chronic Asthma
}

\author{
Meixuan Liu (iD) 1,2 \\ Mengtian Shan' \\ Yunxuan Zhang ${ }^{3}$ \\ Zhongliang Guo (D) 1,2 \\ 'Department of Respiratory Medicine, \\ Shanghai East Hospital, School of \\ Medicine, Tongji University, Shanghai, \\ 200123, People's Republic of China; \\ ${ }^{2}$ Department of Respiratory Medicine, \\ Shanghai East Clinical Medical College, \\ Nanjing Medical University, Shanghai, \\ 200 I23, People's Republic of China; \\ ${ }^{3}$ Department of Pharmacy, Huadong \\ Hospital, Fudan University, Shanghai, \\ 200040, People's Republic of China
}

\begin{abstract}
Purpose: Airway remodeling is an important feature of chronic asthma, and yet there are few effective therapeutic strategies. Progranulin (PGRN) has been shown to have lung protective functions, but the role of PGRN in asthmatic airway remodeling is unclear. We aim to explore the protective potential of PGRN on house dust mite (HDM)-induced airway remodeling and the underlying mechanisms.
\end{abstract}

Methods: In this study, a murine model of chronic asthma was established by HDM sensitization and challenge. Recombinant PGRN was intranasally administrated to mice during the phase of HDM challenge. TGF- $\beta 1$-treated human airway epithelial BEAS-2B cells were utilized to explore the effect of PGRN on airway epithelia exposed to profibrotic conditions and molecular mechanisms.

Results: We found that PGRN treatment attenuated HDM-induced airway remodeling, as evidenced by the suppression of collagen accumulation, mucus overproduction and airway smooth muscle synthesis in HDM-challenged asthmatic mice lungs. Meanwhile, PGRN also reversed the increased levels of autophagy markers and autophagosomes in airway epithelia under mimic asthmatic conditions, thereby controlling the fibrotic process in vivo and in vitro. Specifically, overexpressed HMGB1 and the subsequent RAGE/MAPKs signaling activation due to HDM exposure were abrogated in PGRN-treated asthmatic mice. Furthermore, knockdown of HMGB1 expression significantly restrained the fibrosis formation in TGF- $\beta 1$-induced airway epithelia accompanied by the downregulation of autophagic activity. However, enhancement of extracellular HMGB1 levels blunted the inhibition of autophagic flux by PGRN in airway epithelia, thereby resulting in the augmentation of collagen synthesis and fibrosis.

Conclusion: Taken together, our data revealed that PGRN protected against asthmatic airway remodeling by negatively regulating autophagy via HMGB1 suppression, which might provide new insights into the therapeutic options for HDM-induced chronic asthma.

Keywords: house dust mite-induced asthma, airway remodeling, progranulin, autophagy, HMGB1

\section{Introduction}

Asthma is a chronic inflammatory disease of the airways that is mainly caused by inhaled allergens such as house dust mite (HDM), or other external stimulus, leading to symptoms including cough, wheezing, dyspnea, or chest tightness. ${ }^{1}$ These clinical manifestations of asthma are closely relevant to the structural changes of the airway
Correspondence: Zhongliang Guo

Tel +8618901681968

Email drguozhl@I63.com 
wall, also known as airway remodeling. ${ }^{2}$ Increased airway smooth muscle (ASM) mass, extracellular matrix (ECM) components and collagen deposition, epithelialmesenchymal transition (EMT), reticular basement membrane thickening, and goblet cell metaplasia are identified as the typical pathophysiological traits of airway remodeling in asthma. ${ }^{3}$ Among the complicated mechanisms, TGF$\beta 1$-induced fibrosis of airway walls by promoting matrix accumulation is a key contributor to airway structural remodeling. ${ }^{4}$ Therapeutically, although conventional asthma medications such as inhaled corticosteroid and longacting $\beta 2$-agonists can reduce airway inflammation and hyperresponsiveness, airway fibrosis and remodeling cannot be effectively prevented in chronic asthma. ${ }^{5}$ Therefore, it is necessary to provide a better understanding of the pathogenesis of airway remodeling and explore new beneficial alternatives.

Progranulin (PGRN), a secretory glycoprotein encoded by the GRN gene, is widely expressed in a variety of tissues and cell types, including airway epithelial cells, ${ }^{6}$ macrophages ${ }^{7}$ and microglial cells. ${ }^{8}$ PGRN has pleiotropic properties by participating inflammatory response, regeneration, cell cycle control, and tissue development. ${ }^{9-12}$ Of interest, PGRN is recently regarded as a crucial regulator to prevent fibrotic process in different diseases, such as liver fibrosis, ${ }^{13}$ myocardial infarction ${ }^{14}$ and cutaneous wound healing. ${ }^{15}$ But the effect of PGRN on house dust mite-induced airway remodeling is still elusive. Whether PGRN modulates asthma-related airway fibrosis and what potential mechanisms remain to be elucidated.

Autophagy is a highly conserved catabolic process in which dysfunctional or excessive proteins and organelles are recycled or delivered to maintain cellular homeostasis through the lysosome-mediated degradation pathway. Emerging studies have suggested that autophagy is linked with several inflammatory diseases due to its potential to modulate genetic and immune mechanism. Of note, autophagy dysregulation plays a pivotal role in the process of fibrotic disorders including asthma airway fibrosis. ${ }^{16-18}$ Autophagy pathway activation in the airways from asthmatics is highly associated with the development of airway remodeling. ${ }^{19}$ High mobility group box 1 (HMGB1) is an essential damage-associated molecular pattern (DAMP) protein expressed in various cell types. Extracellular HMGB1 plays critical roles in cell death and injury through acting with the domain receptors, such as receptor for advanced glycation end-products (RAGE) and toll-like receptors (TLRs), leading to mitogen-activated protein kinase (MAPK) and nuclear factor (NF)- $\mathrm{kB}$ activation. ${ }^{20}$ HMGB1 release has been reported to crucially involve in the regulation of autophagy in multiple diseases. ${ }^{21,22}$ Our previous study has found that HMGB1 signaling activation is responsible for HDM-induced airway damage. However, the relationship between HMGB1-related autophagy and airway remodeling in HDM-induced chronic asthma under PGRN treatment is poorly reported to date.

In this study, by using a murine model of HDMinduced chronic asthma in vivo and TGF- $\beta 1$-induced airway epithelial cells in vitro, we sought to explore the roles of PGRN in asthmatic airway remodeling and autophagy, including its mechanism. Our findings demonstrated that PGRN was a protective regimen for asthmatic airway remodeling and fibrosis by modulating autophagy via HMGB1 suppression, indicating PGRN might be a potential therapeutic strategy for HDM-induced asthma.

\section{Materials and Methods}

\section{Animal Model of Chronic Asthma}

Healthy female Balb/c mice at age of 6-8 weeks old (purchased from Changzhou Cavens Laboratory Animal Co. Ltd.) were maintained under specific pathogen-free conditions. All animal protocols were performed in accordance with the National Institutes of Health Guide for the Care and Use of Laboratory Animals. The animal study was reviewed and approved by the Animal Care and Use Committee of Tongii University. To investigate the protective effects of PGRN against HDM-induced airway remodeling, mice were randomly divided into the control group, the HDM group and the HDM+PGRN group ( $\mathrm{n}=8$ /group). The mice in HDM group were sensitized with intraperitoneal (i.p.) injection of 50ug HDM (cat. no. XPB70D3A2.5, Greer Labs, Lenoir, NC, USA) with aluminum hydroxide (Sigma-Aldrich, St. Louis, MO, USA) on days 0, 7 and 14, and then challenged by intranasal (i.n.) administration of 10ug HDM from day 21 three times a week for 4 consecutive weeks. The mice in HDM+PGRN group were intranasally administrated with recombinant PGRN (ProSpec, Rehovot, Israel) ( $2 \mu \mathrm{g}$ dissolved in 20ul PBS) 30 min prior to each inhalation of HDM during challenge phase. The mice in the control group were just treated with PBS instead of HDM or PGRN. All mice were sacrificed $24 \mathrm{~h}$ after the last challenge. The bronchoalveolar lavage fluid (BALF) and lung tissues were collected to analyze inflammatory cells counts and histopathological features, respectively. 


\section{Cell Culture and Treatments}

Human airway epithelial cell line BEAS-2B was purchased from Cell Bank of the Chinese Academy of Sciences (Shanghai, China). Cells were cultured in DMEM (Hyclone, Camarillo, CA, USA) containing 10\% fetal bovine serum (Gibco, Grand Island, NY, USA), 1\% penicillin-streptomycin (Gibco) at $37^{\circ} \mathrm{C}$ in $5 \% \mathrm{CO} 2$. To identify the effect of PGRN on airway fibrosis, the cells were pretreated with recombinant PGRN, and then (2 hours later) incubated with 10ng/mL TGF- $\beta 1$ (Peprotech, Rocky Hill, NJ, USA) for another $24 \mathrm{~h}$ or $48 \mathrm{~h}$ respectively. Phosphate-buffered saline (PBS) was used as negative control.

\section{Cell Transfection}

The siRNAs targeting HMGB1 gene (siRNA-HMGB1) and siRNA-NC were purchased from GenePharma Company (Shanghai, China). BEAS-2B cells were planted in 6-well $\left(2 \times 10^{5}\right)$ and cultured overnight before transfection. Then, the cells were subjected to the transient transfection by the Lipofectamine 2000 (Invitrogen, Camarillo CA, USA) according to the manufacturer's instructions. All treated BEAS-2B cells were collected for further different experiments.

\section{Analysis of Lung Morphology}

Lung tissue samples were in $4 \%$ formaldehyde and then embedded in paraffin. The tissue sections (5um) were stained with haematoxylin and eosin (H\&E), periodic acid Schiff (PAS) and Masson's trichrome. The images were obtained by a light microscope (Olympus X73, Tokyo, Japan). The lung inflammation score was calculated as previously described. ${ }^{23}$

\section{Western Blot Analysis}

Lung tissues or cells were washed by PBS and harvested with RIPA. The protein lysates were electrophoresed in SDS-PAGE gel and then transferred onto PVDF membranes. After blocked with 5\% BSA, the membranes were incubated overnight at $4^{\circ} \mathrm{C}$ with primary antibodies against $\alpha$-smooth muscle actin ( $\alpha$-SMA) (1:1000; ab21027), Collagen-I (1:1000; ab260043) collagen-III (1:1000; ab184993), RAGE (1:1000; ab216329), p62 (1:1000; ab56416) (Abcam, Cambridge, MA, USA), LC3 $(1: 1000 ; 4108 S)$, HMGB1 $(1: 1000 ; 6893)$, phospho(p)JNK (1:1000; 4668), JNK (1:1000; 9252), p-p38 (1:1000; 9211), p38 (1:1000; 9212), p-ERK1/2 (1:1000; 9101),
ERK1/2 (1:1000; 8544) (Cell Signaling Technology, Danvers, MA), Beclin-1 (1:1000; 11306) (Proteintech, Rosemont, IL, USA) and GAPDH (1:1000; 5174) (Cell Signaling Technology). Then the membranes were rinsed in TBST and incubated with HRP-conjugated anti-rabbit secondary antibodies for $2 \mathrm{~h}$ at room temperature. ECL system was used to detect the protein blots.

\section{Immunohistochemical Staining}

Lung tissues were fixed with 4\% paraformaldehyde and incubated with primary antibodies against $\alpha$-SMA (1:500; ab32575), Collagen-I (1:500; ab34710), Beclin-1 (1:500; ab62557) (Abcam) or LC3 (1:500; 3868) (Cell Signaling Technology) overnight at $4^{\circ} \mathrm{C}$, and then incubated with according secondary antibodies for $1 \mathrm{~h}$ at room temperature. Finally, the slides were counterstained with hematoxylin. The captured images were analyzed using an Image Pro Plus 4.5

\section{Immunofluorescence Staining}

The immunofluorescence staining was performed to detect the expression levels of indicated proteins in cells and lung tissues. Briefly, BEAS-2B cells were incubated with the primary anti- $\alpha$-SMA antibody $(1: 300 ;$ ab32575) (Abcam) or anti-LC3 antibody (1:500; 18725-1-AP) (Proteintech) overnight $\left(4^{\circ} \mathrm{C}\right)$. Followed by washing with PBS, the samples were stained with goat anti-rabbit fluorophoreconjugated secondary antibodies for $1 \mathrm{~h}$ at room temperature. The nuclei were counterstained with DAPI. The images were obtained by Leica fluorescence microscope.

\section{Transmission Electron Microscopy (TEM)} Cells from different groups were prefixed with 2.5\% ice-cold glutaraldehyde for $1 \mathrm{~h}$. After rinsed by PBS, the samples were pro-fixed with $1 \% \mathrm{OsO} 4$ and embedded in pure acetone and resin embedding fluid (2:1). Then ultra-thin sections $(50-60 \mathrm{~nm})$ were double stained with 4\% uranyl acetate and lead citrate. Transmission electron microscopy was applied to detect the formation of autophagosomes in airway epithelial cells.

\section{mRFP-GEP-LC3 Adenovirus Transduction}

An mRFP-GEP-LC3 dual-fluorescence assay was performed to determine the autophagic flux. Briefly, when the cells reached nearly $60 \%$ confluence, they were transfected with mRFP-GEP-LC3 adenovirus (Genomeditech, Shanghai, China) prior to the indicated 
experiments. The cells images were captured to demonstrate the autophagic flow by a confocal microscope (Olympus FV 1000, Tokyo, Japan).

Procedures for the measurements of quantitative reverse transcription PCR (qRT-PCR), flow cytometric analysis and enzyme-linked immunosorbent assay (ELISA) are presented as online Supplemental Materials.

\section{Statistical Analysis}

Data were presented as mean \pm standard error of the mean (SEM). Multiple groups' comparisons were performed using one-way ANOVA with the Bonferroni's post hoc test. Statistical analysis was conducted using SPSS19.0. $\quad \mathrm{P}<0.05$ was considered as statistically significant.

\section{Results}

\section{PGRN Administration Mitigates} HDM-Induced Airway Inflammation and Remodeling in Mice

To determine the function of PGRN in allergic airway inflammation and remodeling, a HDM-induced murine asthma model was utilized (Figure 1A). Compared to the control group, HDM-challenged asthmatic mice had a remarkable enhancement in inflammatory cells infiltration and airway wall thicken, while PGRN administration effectively alleviated these histopathological changes in asthmatic mice (Figure 1B and C). Accordingly, lung inflammation score was also lower in HDM+PGRN group than those in HDM alone group (Figure 1D).
A

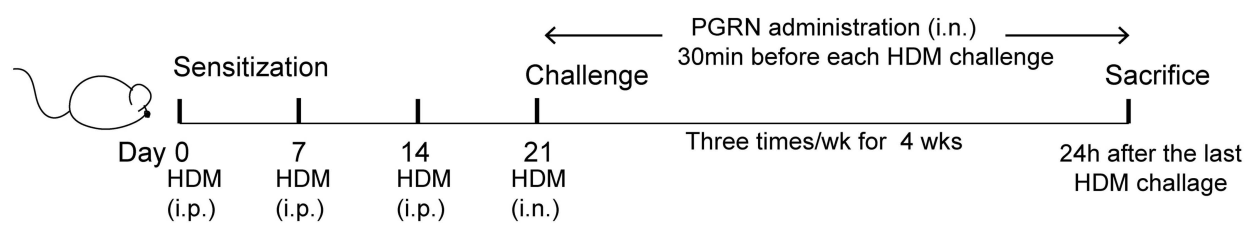

B

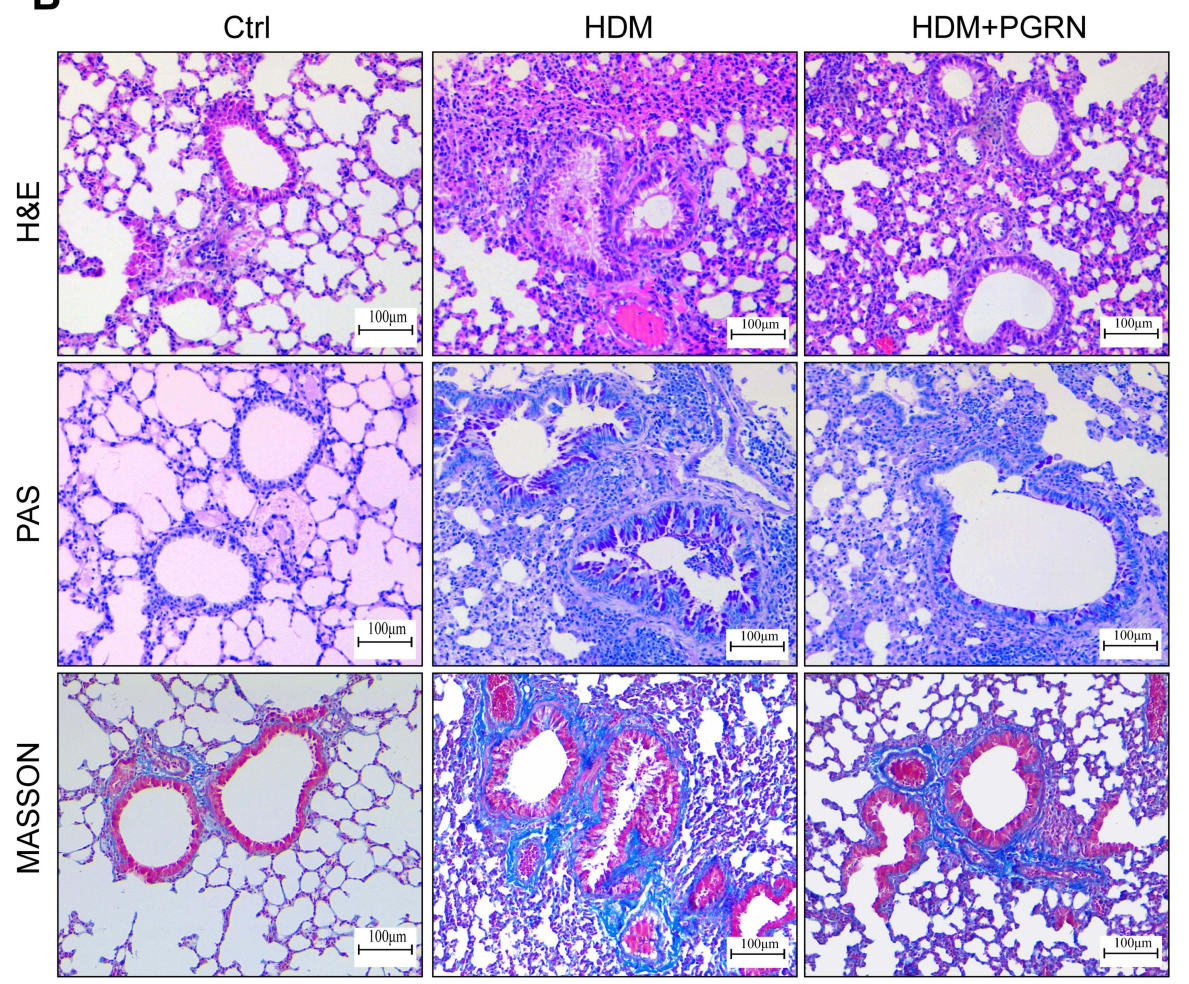

C

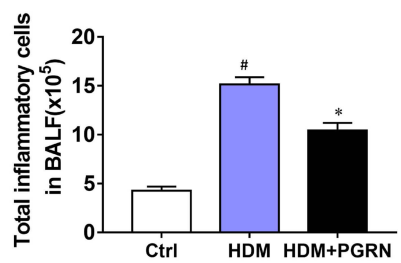

D

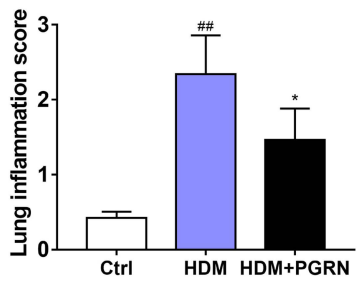

$\mathbf{E}$

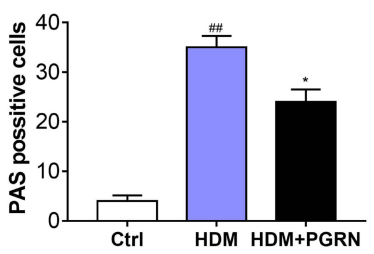

$\mathbf{F}$

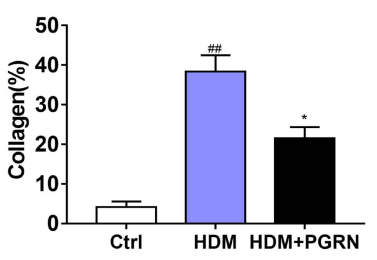

Figure I Effects of PGRN on airway remodeling in HDM-challenged asthmatic mice. (A) Schematic diagram of the experimental plan for HDM-induced murine asthma. (B) H\&E, PAS and Masson's trichrome staining of lung sections from different groups. (C) The counts of total inflammatory cells in BALF from mice analyzed by Wright staining. (D) Levels of inflamed lung areas in mice. (E) Quantification of PAS-positive mucous cells in airways of each group. (F) Analysis of collagen deposition in airway tissues. Quantitation of lung morphology analysis was based on 5 random areas from each sample. Data are expressed as means \pm SEMs of three independent experiments with four mice per group. ${ }^{\#} \mathrm{p}<0.05$ vs control group, ${ }^{\#} \mathrm{p}<0.01$ vs control group, ${ }^{*} \mathrm{p}<0.05$ vs HDM group. 
Compared with HDM+PGRN group, HDM-challenged mice had a stronger PAS-positive staining in airway tissues which represented mucus overproduction and goblet cell hyperplasia (Figure 1B and E). Additionally, in contrast to the control group, Masson's trichrome staining showed that collagen deposition and airway wall thickness were obviously increased in HDM-induced asthmatic mice, which were significantly inhibited by PGRN treatment (Figure 1B and F). For another, PGRN effectively reduced HDM-mediated Th2-type allergic airway inflammation (Supplementary Figure S1A-C). Since airway remodeling is characterized by myofibroblast differentiation, subepithelial fibrosis, and increased ECM deposition, we further assessed the expression of fibrosis-related markers $\alpha$-SMA and Collagen-I in lung tissues by immunochemistry analysis. Our findings showed that $\alpha$-SMA and Collagen-I were markedly expressed in peribronchial tissues of asthmatic mice in contrast to those in the control group. However, compared with the HDM group, the expression of $\alpha$-SMA and Collagen-I was significantly decreased in the HDM+PGRN group (Figure 2A-C). Meanwhile, the elevated mRNA levels of fibrosis-related genes $\alpha$-SMA, Collagen-I, TGF- $\beta 1$ and MMP9 (Figure 2D-G) in HDM-challenged mice were markedly repressed by PGRN treatment. Collectively, the results indicated that PGRN had an ability to attenuate airway inflammation and remodeling in HDM-induced asthma model.

\section{PGRN Suppresses Fibrotic Process in TGF- $\beta I$-Treated Human Airway Epithelial Cells}

To identify the effect of PGRN on airway epithelia under the mimic fibrotic conditions in vitro, human airway epithelial BEAS-2B cells were treated with variable concentrations PGRN for $24 \mathrm{~h}$ under the induction of $10 \mathrm{ng} / \mathrm{mL}$ TGF- $\beta 1$, a known profibrotic factor to induce myofibroblast differentiation. Our results showed that PGRN $(20,50$, or $100 \mathrm{ng} / \mathrm{mL})$ dose-dependently inhibited the expression of TGF- $\beta 1$-induced fibrosis markers $\alpha$-SMA, Collagen-I and Collagen-III. (Supplementary Figure S2A-D). BEAS-2B cells exposed to PGRN also revealed the mRNA upregulation of EMT biomarker E-cadherin accompanied by the reduction of $\mathrm{N}$-cadherin (Supplementary Figure S2E). Then, BEAS-2B cells were incubated with TGF- $\beta 1$ for different time duration under $100 \mathrm{ng} / \mathrm{mL}$
A
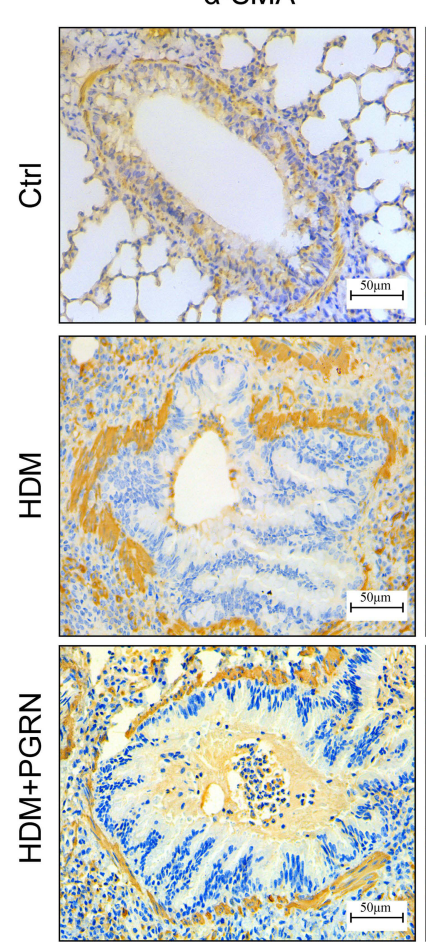

Collagen-I
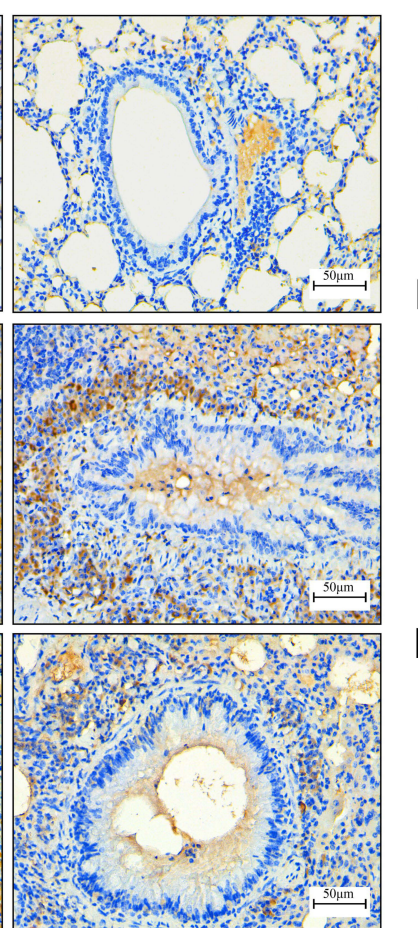

B

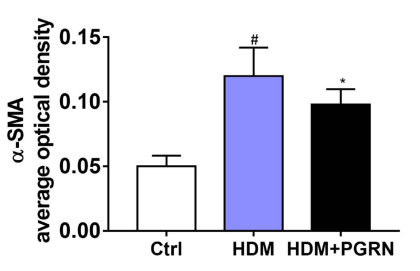

D

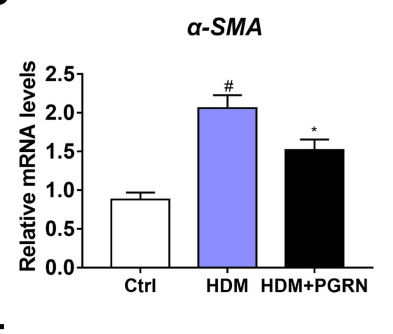

$F$

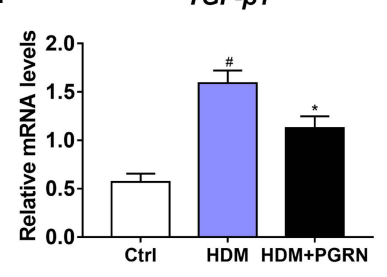

C

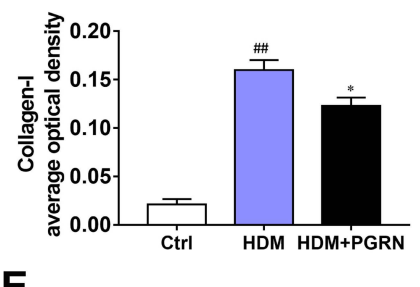

E

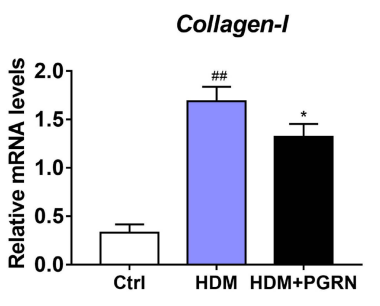

G

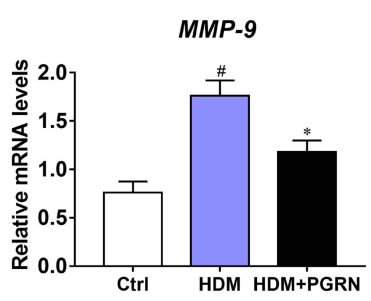

Figure 2 PGRN restrains HDM-induced collagen synthesis and fibrosis in mice. (A) Protein expression of $\alpha$-SMA and collagen-I in mice was detected by immunohistochemical staining. (B and $\mathbf{C}$ ) $\alpha$-SMA and collagen-I expression levels according to average optical density. The relative mRNA expression of fibrosis-related genes including $\alpha$ SMA (D), collagen-I (E), TGF- $\beta$ I (F), MMP-9 (G) were measured by qRT-PCR. For immunochemistry assay, results are obtained from 5 random areas of each sample. Data are expressed as means \pm SEMs of three independent experiments with four mice per group. ${ }^{\#} p<0.05$ vs control group, ${ }^{\# \#} p<0.01$ vs control group, ${ }^{*} p<0.05$ vs HDM group. 
PGRN treatment or not. As illustrated in Figure 3A and B, compared with the controls, the expression of fibrosis-related markers $\alpha$-SMA, Collagen-I and Collagen-III was dramatically upregulated in TGF- $\beta 1$-treated cells in a time-dependent manner. Meanwhile, PGRN treatment decreased the protein levels of these markers evoked by TGF- $\beta 1$ stimulation. Furthermore, immunofluorescence staining was applied to assess $\alpha$-SMA expression in BEAS-2B cells with different treatments. Compared to the control, $\alpha$-SMA positive cells were apparently increased in response to TGF- $\beta 1$ exposure, especially in the $48 \mathrm{~h}$ TGF- $\beta 1$-treated group. However, TGF- $\beta 1$-induced $\alpha$ SMA overexpression was partly reduced in the cells with PGRN treatment (Figure 3C and D). These data suggested that PGRN could restrain TGF- $\beta 1$-mediated fibrosis by decreasing the expression of ECM components in airway epithelia.

\section{PGRN Downregulates Autophagic Activity in Asthmatic Mice and Human BEAS-2B Cells}

In order to evaluate whether PGRN could regulate autophagy associated with asthmatic airway remodeling and fibrosis, we further examined the expression of acknowledged autophagy markers LC3 and Beclin-1 in different mice lungs. Immunochemistry analysis revealed that LC3 and Beclin-1 were highly expressed in the airways of HDM-challenged asthmatic mice. Few staining with anti-LC3 and anti-Beclin -1 from control mice were observed. Compared with HDM group, the asthmatic mice receiving PGRN administration had moderate expression of the two autophagy markers in airway tissues (Figure 4A-C). In addition, the degree of Beclin-1 and LC3 expression was concomitant with the pathological changes of airway tissues in the mice. In vitro, for autophagy
A

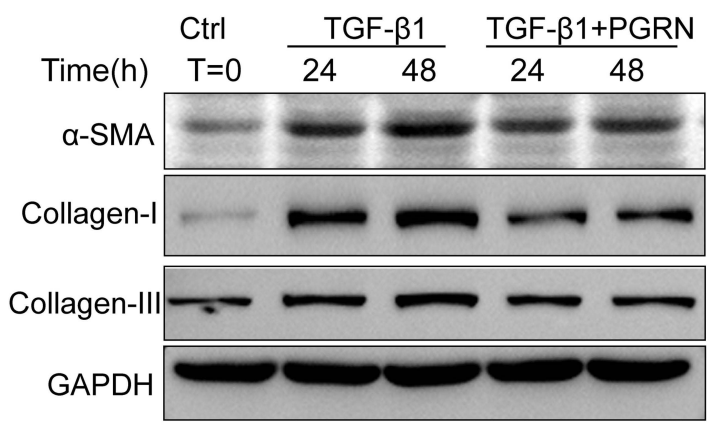

B

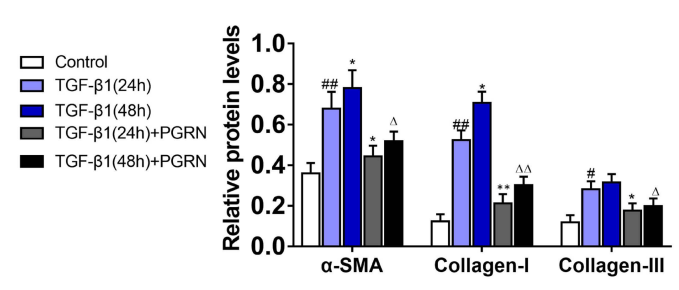

D

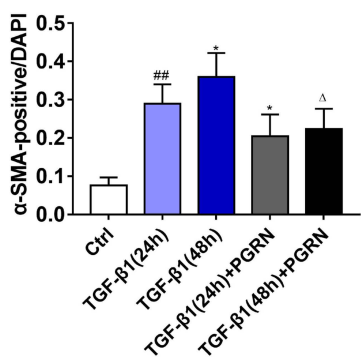

C
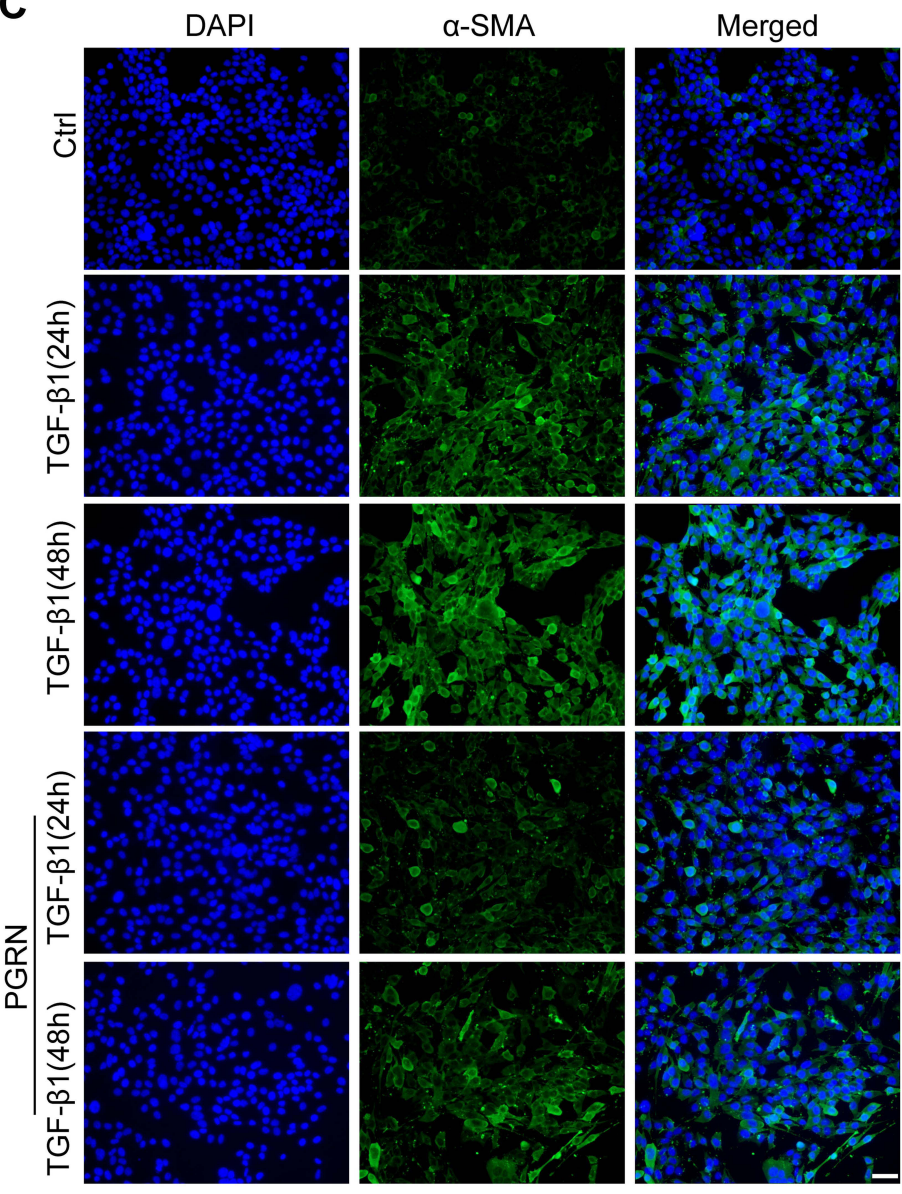

Figure 3 PGRN inhibits TGF- $\beta$ I-mediated fibrotic process in BEAS-2B cells. (A) After the induction by $48 \mathrm{~h}$ or $72 \mathrm{~h}$ TGF- $\beta$ I (IOng/mL) with or without PGRN, Western blotting was performed to detect the expression of $\alpha$-SMA, collagen-I and collagen-III. (B) Grey values of the indicated proteins were shown as bar graphs. (C) $\alpha$-SMA expression (green) was detected by immunofluorescence assay in BEAS-2B cells under different treatments. (D) The ratio of $\alpha$-SMA positive cells from different groups. Data are expressed as means \pm SEM of three independent experiments. ${ }^{\#} \mathrm{p}<0.05$ vs control group, ${ }^{\#} \mathrm{p}<0.05$ vs control group, ${ }^{\#} \mathrm{p}<0.0 \mathrm{l}$ vs control group, ${ }^{*} \mathrm{p}<0.05$ vs TGF- $\beta$ I ( $24 \mathrm{~h}$ ) group, ${ }^{* *} \mathrm{p}<0.0$ I vs TGF- $\beta$ I (48h) group, ${ }^{\Delta} \mathrm{p}<0.05$ vs TGF- $\beta$ I (48h) group, ${ }^{\Delta \Delta} \mathrm{p}<0.0$ I vs TGF- $\beta$ (48h) group. 
assay, the ultrastructural assessment of epithelial cells was analyzed by TEM. Figure 4D and E displayed that PGRN treatment reversed the increased numbers of autophagosomes in BEAS-2B cells under 48h TGF- $\beta 1$ induction. In parallel, we found that increased apoptotic cell death following TGF- $\beta 1$ treatment was also restrained by PGRN (Figure 4F and G). These findings confirm the negative regulation of PGRN on autophagy which takes part in asthmatic fibrosis and airway remodeling.

\section{PGRN Blocks HDM-Induced HMGBI Expression and the Downstream RAGE-MAPKs Pathways in Mice}

Previous studies have verified that HMGB1 plays a role in the modulation of autophagy as an essential DAMP molecular. $^{24,25}$ To explore the key role of HMGB1 in asthma-related autophagy and whether inhibition of autophagy by PGRN is linked with HMGB1-mediated signaling, we evaluated HMGB1 expression and its potential signaling pathway in mice lungs under different groups. As expected, the protein levels of HMGB1 in HDMchallenged asthmatic mice lungs were significantly higher than those in the control group, whereas markedly diminished in PGRN-treated mice. Of note, RAGE is believed as a usual kind of pattern recognition receptors (PRRs) binding HMGB1. HMGB1/RAGE axis is critically implicated in the modulation of inflammation, autophagy and fibrosis via the downstream MAPKs pathway in asthma pathogenesis. $^{26}$ To further test the possible signaling transduction of HMGB1 in PGRN-treated asthmatic mice, we then examined the changes of RAGE, JNK, ERK1/2 and p38. The immunoblotting analysis showed that upregulation of RAGE expression triggered by HDM was inhibited
A

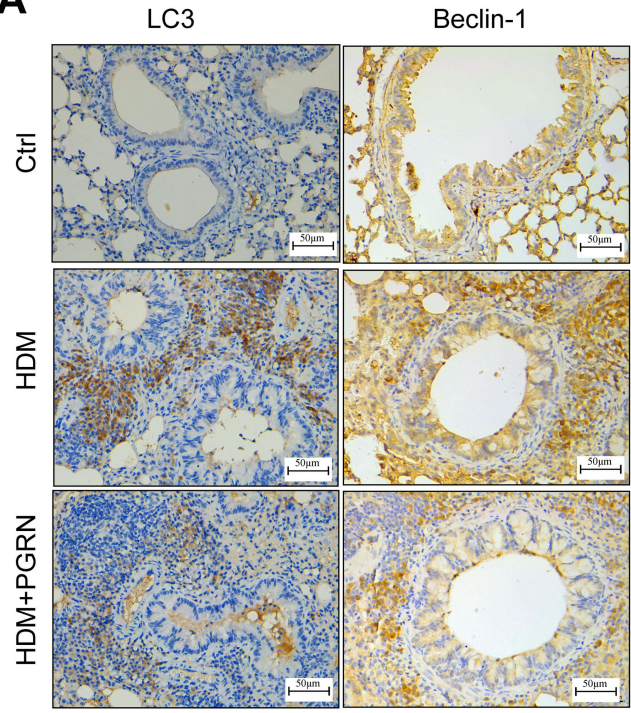

B

D

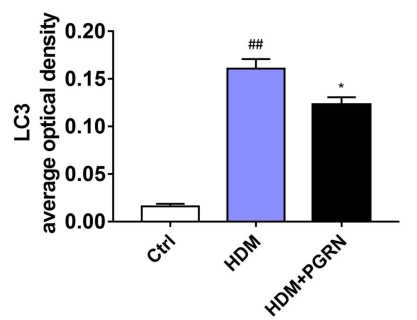

C

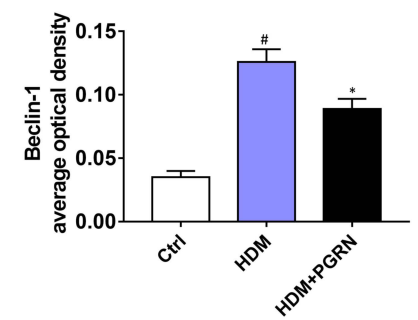

Ctrl

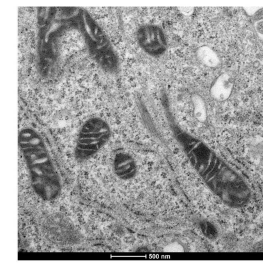

TGF- $\beta 1$ (48h)

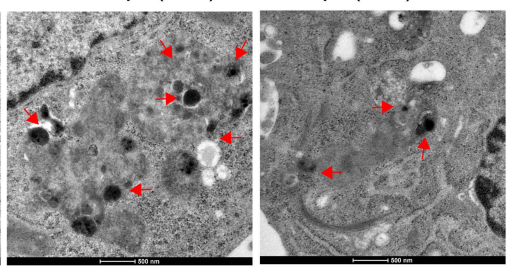

E

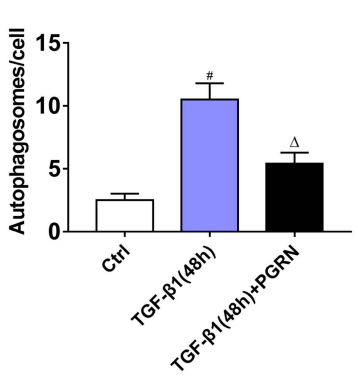

$\mathbf{F}$

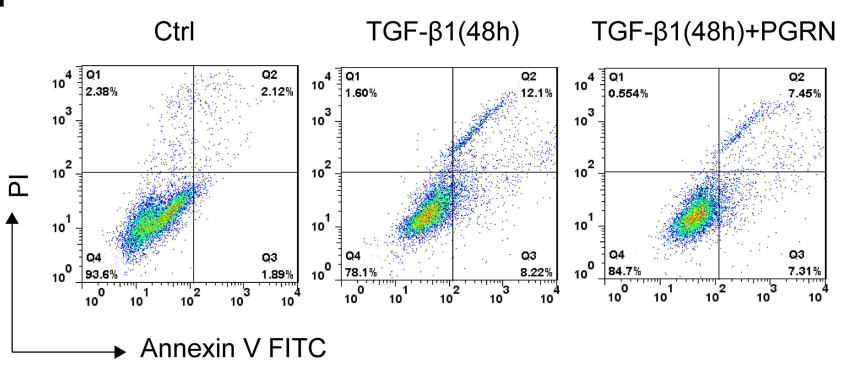

G

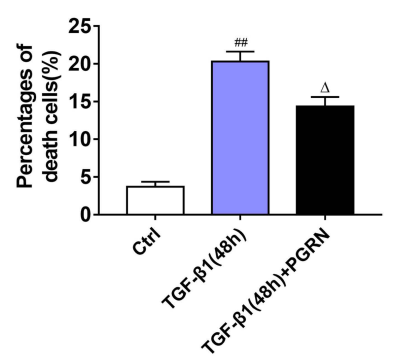

Figure 4 PGRN reduces airway autophagy in asthmatic mice and TGF- $\beta$ I-treated BEAS-2B cells. (A) LC3 and Beclin-I expression in airway tissues of mice was assessed by immunohistochemistry. (B and C) Semi-quantitative analysis showed that PGRN significantly decreased the expression levels of LC3 and Beclin-I in mice exposed to HDM. (D) Representative TEM images of autophagosomes (red arrows) in TGF- $\beta$ I-induced BEAS-2B cells with PGRN treatment or not. Scale bar=500nm. (E) Quantification of the numbers of autophagosomes in cells. (F) Apoptosis of cells was measured by the FITC-annexin V/PI assay. (G) Flow cytometry indicated that PGRN reduced apoptotic cell death in TGF- $\beta$ I-treated cells. For analysis of immunochemistry assay, results are pooled from 5 random areas with four mice per group. Data are expressed as means \pm SEM of three independent experiments. ${ }^{\#} \mathrm{p}<0.05$ vs control group, ${ }^{\#} \mathrm{p}<0.0$ I vs control group, ${ }^{*} \mathrm{p}<0.05$ vs HDM group, ${ }^{\Delta} \mathrm{p}<0.05$ vs TGF- $\beta$ I (48h) group. 
A

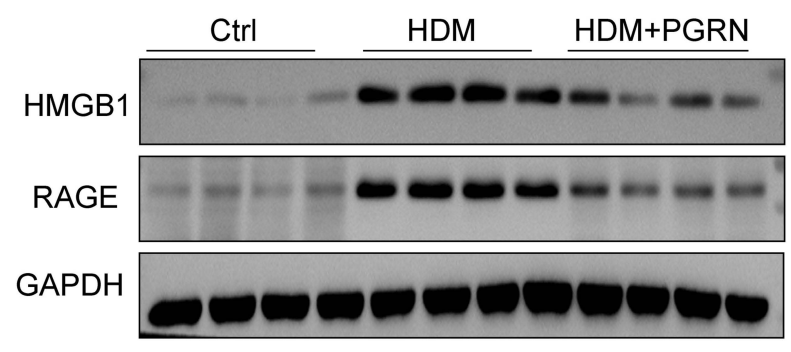

C

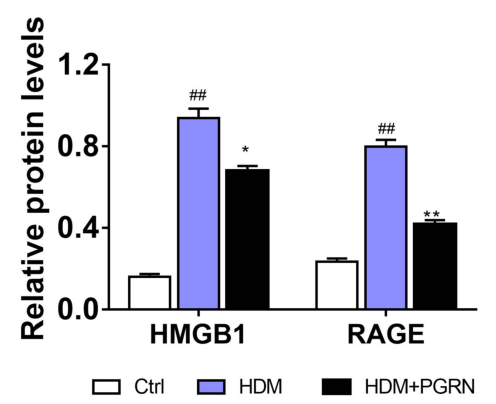

B

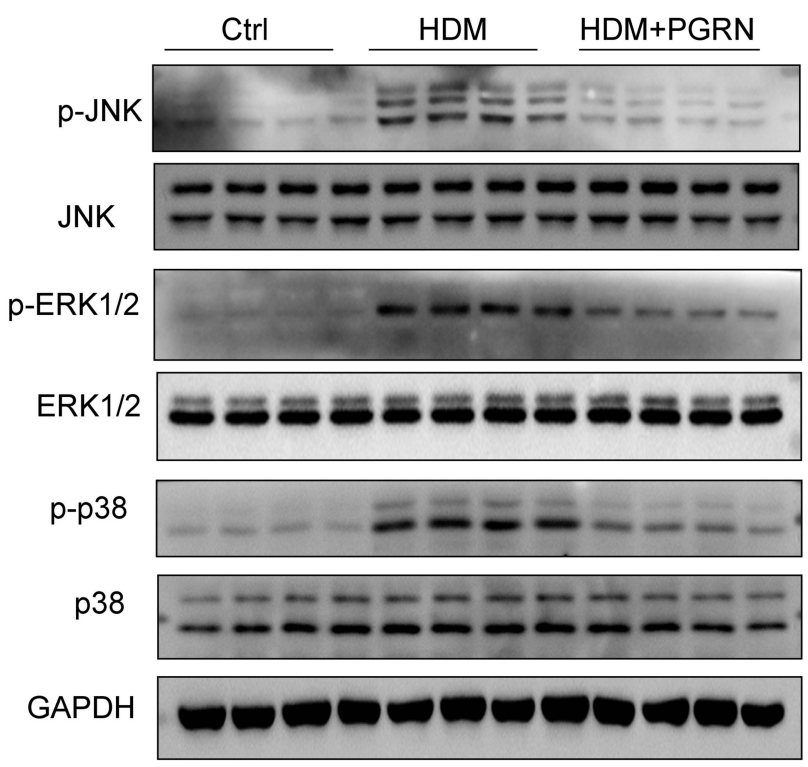

D

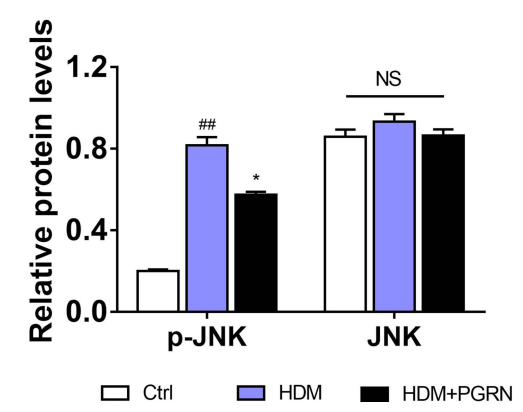

E

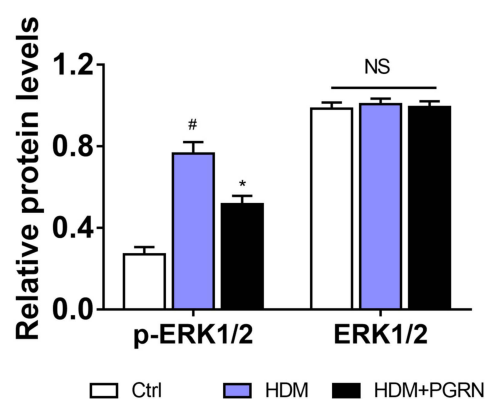

$\mathbf{F}$

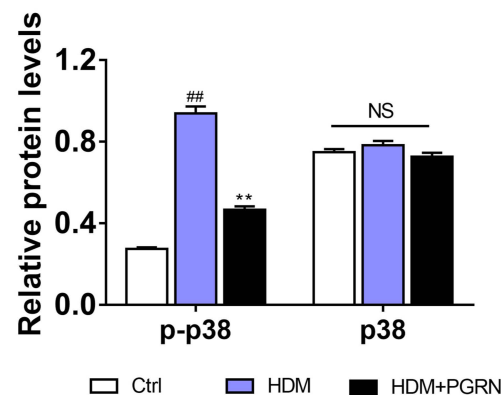

Figure 5 Effect of PGRN on HMGBI, RAGE and MAPK pathways in HDM-challenged mice. (A) PGRN downregulated HMGBI and RAGE protein expression in mice lungs upon HDM exposure. (B) The effect of PGRN on phosphorylated and total JNK, ERKI/2, and p38 expression were evaluated by immunoblotting analysis. (C-F) Quantitative densitometric ratio of the above proteins normalized to GAPDH in each group. For immunoblotting analysis, data are expressed as means \pm SEM of three independent experiments with four mice per group. ${ }^{\#}<<0.05$ vs control group, ${ }^{\#} p<0.01$ vs control group, ${ }^{*} p<0.05$ vs HDM group, ${ }^{* *} \mathrm{p}<0.0$ I vs $\mathrm{HDM}$ group.

after PGRN treatment. HDM-elicited phosphorylations of JNK, ERK1/2 and p38 were obviously decreased in the PGRN-treated asthmatic mice (Figure 5). These results indicated that PGRN treatment blocked HMGB1 expression and its downstream signaling during HDM-induced allergic asthma.

\section{HMGBI is Required for the Suppressive Effect of PGRN on Autophagy Under Fibrotic Conditions}

To ascertain whether HMGB1 is involved in the antifibrosis functions of PGRN on airway epithelia, we first deleted HMGB1 via siRNA. After knockdown of HMGB1 expression, the BEAS-2B cells were exposure to $10 \mathrm{ng} / \mathrm{mL}$ TGF- $\beta 1$ for $48 \mathrm{~h}$ with or without $100 \mathrm{ng} / \mathrm{mL}$ PGRN treatment. We first evaluated the role of HMGB1 in autophagy of airway epithelia following TGF- $\beta 1$ exposure. The immunoblotting analysis revealed that HMGB1 deficiency crucially restrained autophagy activation, shown as the downregulation of autophagy proteins LC3, Beclin-1 and p62, and further suppressed overexpression of $\alpha$-SMA, collagen-I and collagen-III induced by TGF- $\beta 1$ (Figure 6A and B). Moreover, the double immunofluorescent staining was used to assess the degree of fibrosis together with autophagic activity. As illustrated in Figure 6C and D, the staining intensity of dual-labeled LC3 (red) and $\alpha$-SMA (green) was substantially decreased in the TGF- $\beta 1+\mathrm{PGRN}$ co-culture groups compared with TGF- $\beta 1$-treated alone groups, which were in line with our in vivo results. Particularly, in the presence of PGRN 
A

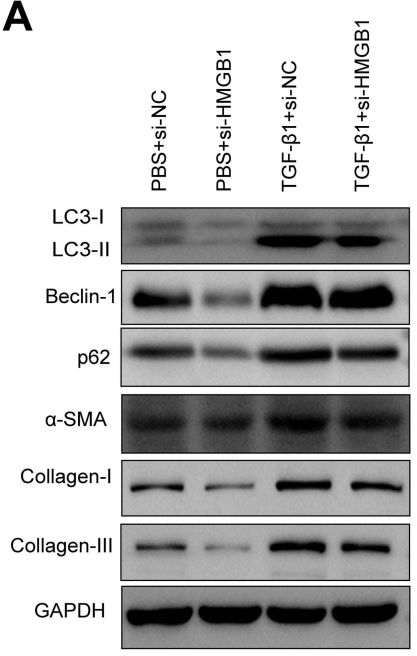

B

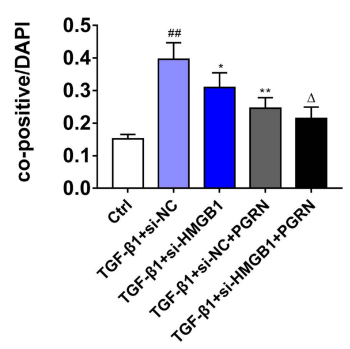

C

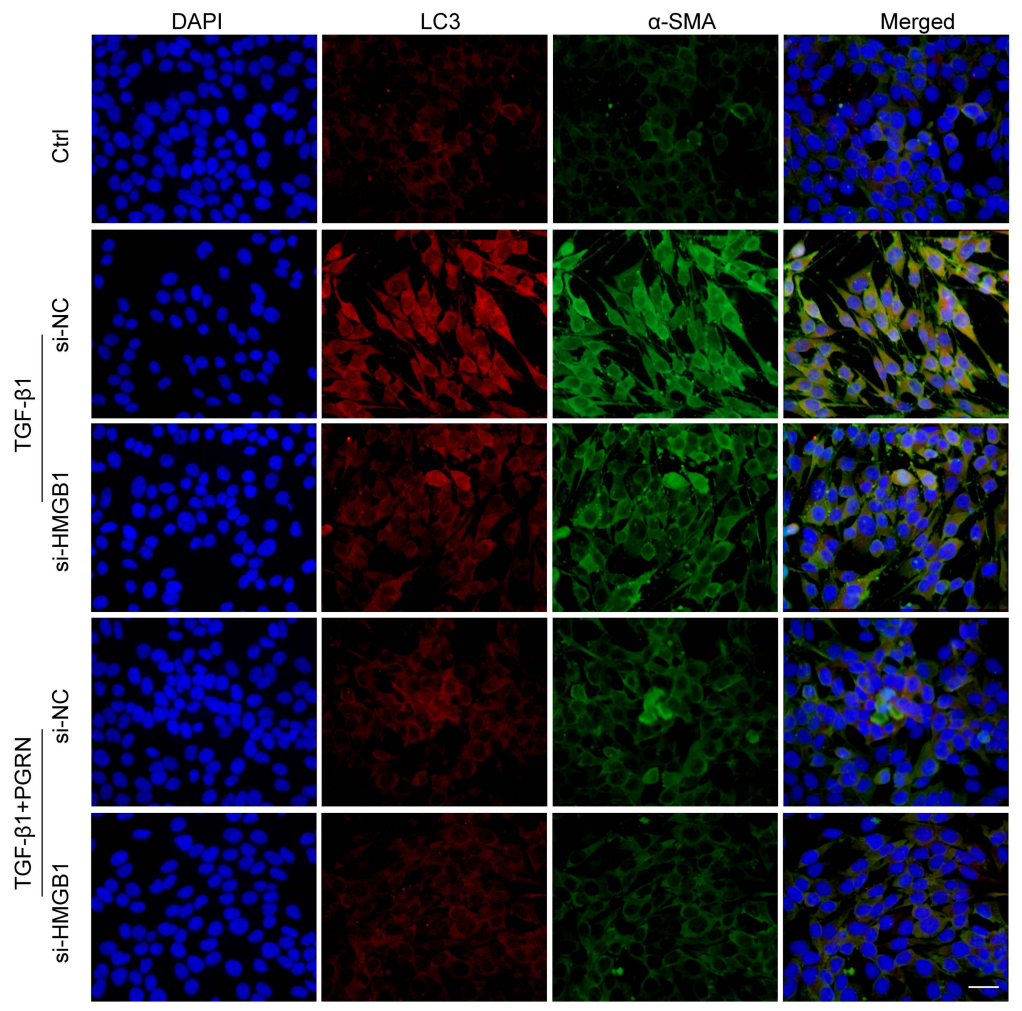

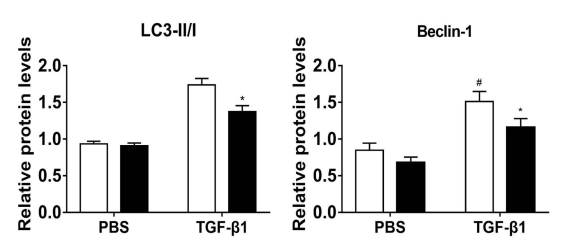
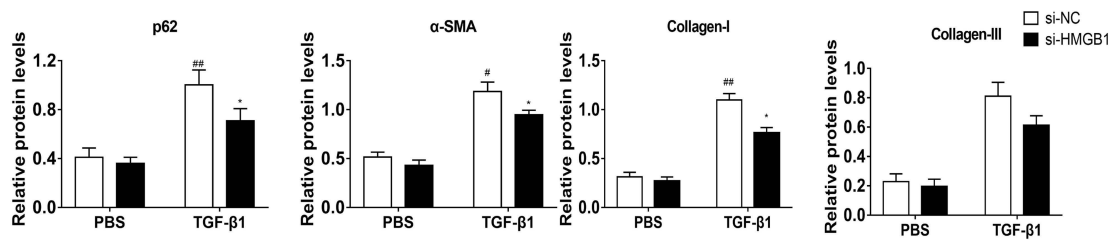

Figure $6 \mathrm{HMGBI}$ is responsible for the negative modulation of PGRN on autophagy under fibrotic conditions.(A) After silencing HMGBI, the expressions of autophagy (LC3, p62, Beclin-I) and fibrosis markers ( $\alpha$-SMA, collagen-I, collagen-III) in BEAS-2B cells were measured by Western blot. (B) Greyscale analysis of the above proteins. (C) Double immunofluorescence staining with LC3 and $\alpha$-SMA in TGF- $\beta$ I-treated BEAS-2B cells transfected with si-HMGBI or si-NC in the presence or absence of PGRN. (D) Co-dying data (LC3 and $\alpha$-SMA) was summarized as bar graph. Scale Bar $=100 \mu \mathrm{m}$. Data are expressed as means \pm SEM of three independent experiments. ${ }^{*}<<0.05$ vs control group, ${ }^{\#} \mathrm{p}<0.01$ vs control group, ${ }^{*} \mathrm{p}<0.05$ vs TGF- $\beta \mathrm{I}+$ si-NC group, ${ }^{* *} \mathrm{p}<0.01$ vs TGF- $\beta \mathrm{I}+\mathrm{si}-\mathrm{NC}$ group, ${ }^{\Delta} \mathrm{p}<0.05$ vs TGF- $\beta$ I+si-HMGBI group.

treatment, the cells transfected with si-HMGB1 had weaker co-staining of LC3 and $\alpha$-SMA elicited by TGF$\beta 1$ stimulation than the si-NC group. In this regard, HMGB1 contributed to TGF- $\beta 1$-mediated fibrosis as a possible target of PGRN. To further clarify the relationship between HMGB1, PGRN, fibrosis and autophagy, we pretreated BEAS-2B cells with 500ng/mL recombinant HMGB1 (rHMGB1) (Sigma-Aldrich, Stockholm, Sweden) or PBS vehicle for $12 \mathrm{~h}$ prior to treatment with TGF- $\beta 1$ in the presence or the absence of PGRN. Under the same fibrotic conditions, BEAS-2B cells were additionally subjected to $5 \mathrm{mM}$ autophagy inhibitor 3-methyladenine (3-MA) (Sigma-Aldrich, St. Louis, USA). As expected, PGRN treatment reversed the increased expression of autophagy proteins, thereby reducing collagen accumulation and fibrosis, which was similar with the results under 3-MA treatment. Nevertheless, rHMGB1 pretreatment aggravated autophagic activity in airway epithelia exposed to TGF- $\beta 1$, thus facilitating the fibrotic process. In addition, the negative effects of PGRN on autophagy and fibrosis upon TGF- $\beta 1$ insult were obviously blunted in the cells pretreated with rHMGB1 (Figure 7A and B). Meanwhile, PGRN-exerted inhibitory effect on autophagy flux was significantly reversed by rHMGB1 treatment (Figure 7C and D). Correspondingly, the decreased counts of autophagosomes as well as apoptotic cell death in PGRN-treated cells were also compromised after rHMGB1 treatment (Supplementary Figure S3A and 


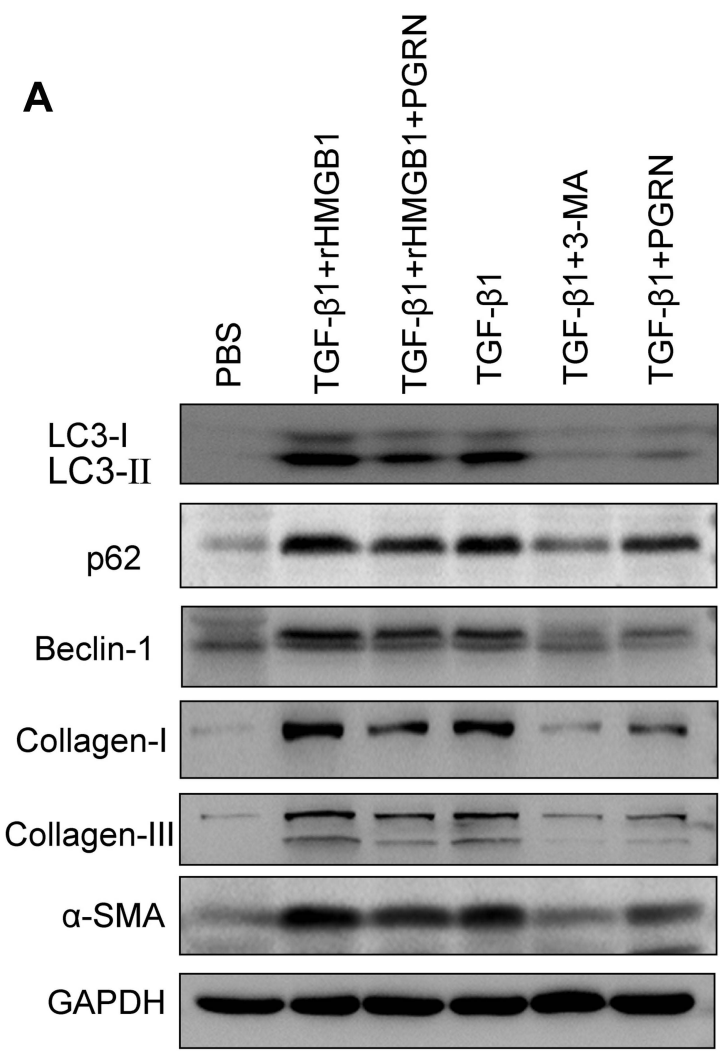

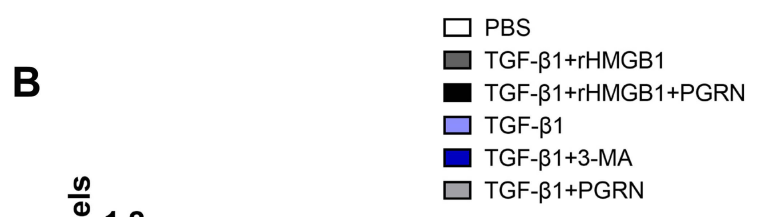
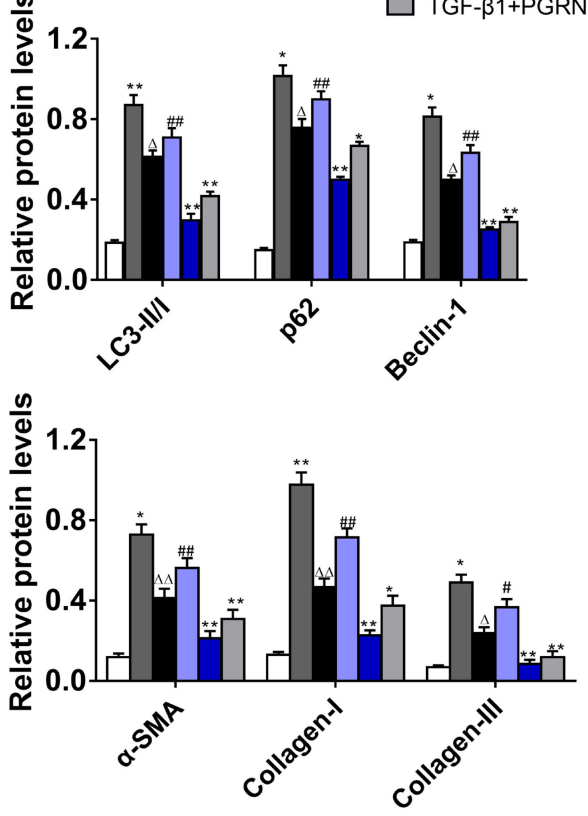

D

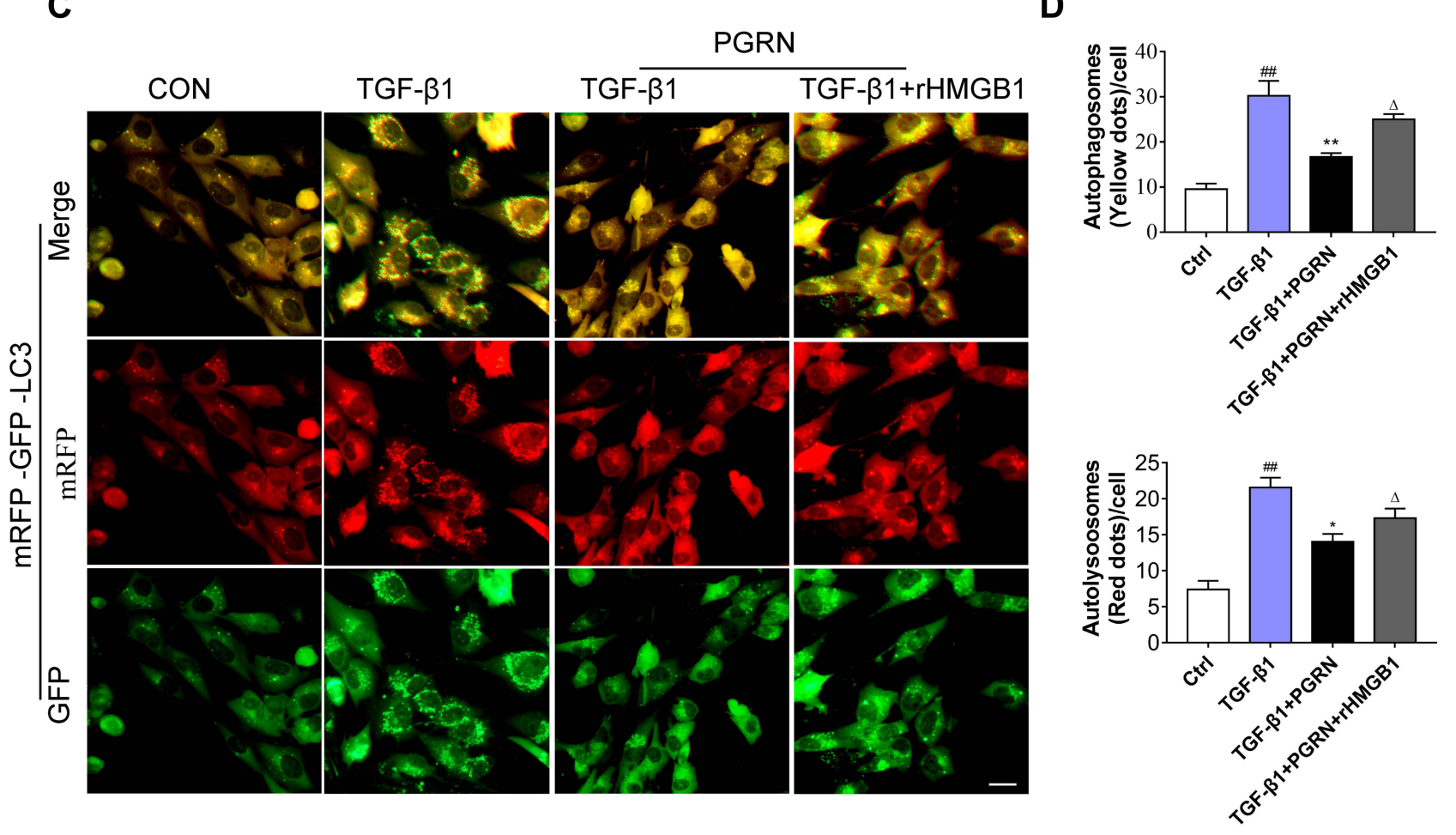

Figure 7 Increased extracellular HMGBI offsets the inhibition of PGRN on TGF- $\beta$ I-driven fibrosis through facilitating autophagy. (A) Immunoblotting analysis showed that PGRN or autophagy inhibitor 3-MA restrained expression of autophagy and fibrosis markers in BEAS-2B cells upon TGF- $\beta$ I exposure, while enhancement of extracellular HMGBI by rHMGBI pretreatment overturned this effect. (B) Relative densitometry of the indicated proteins to GAPDH was displayed as statistical graphs. (C) Representative images of mRFP-GFP-LC3 dual-fluorescence assay showed autophagic flux in BEAS-2B cells with different treatments. Scale bar= 20 $\mu \mathrm{m}$. (D) Quantification of the counts of autophagosomes (yellow dots) and autolysosomes (red dots) in merged images. Data are expressed as means \pm SEM of three independent experiments. ${ }^{\#}<0.05$ vs control group, ${ }^{\#} \mathrm{P}<0.0 \mathrm{I}$ vs control group, ${ }^{*} \mathrm{p}<0.05$ vs TGF- $\beta$ I group, ${ }^{*}{ }^{*} \mathrm{p}<0.01$ vs TGF- $\beta$ I group, ${ }^{\Delta} \mathrm{p}<0.01$ vs TGF- $\beta$ I $+\mathrm{PGRN}$ group, ${ }^{\Delta \Delta} \mathrm{p}<0.01$ vs TGF- $\beta$ I +PGRN group. 


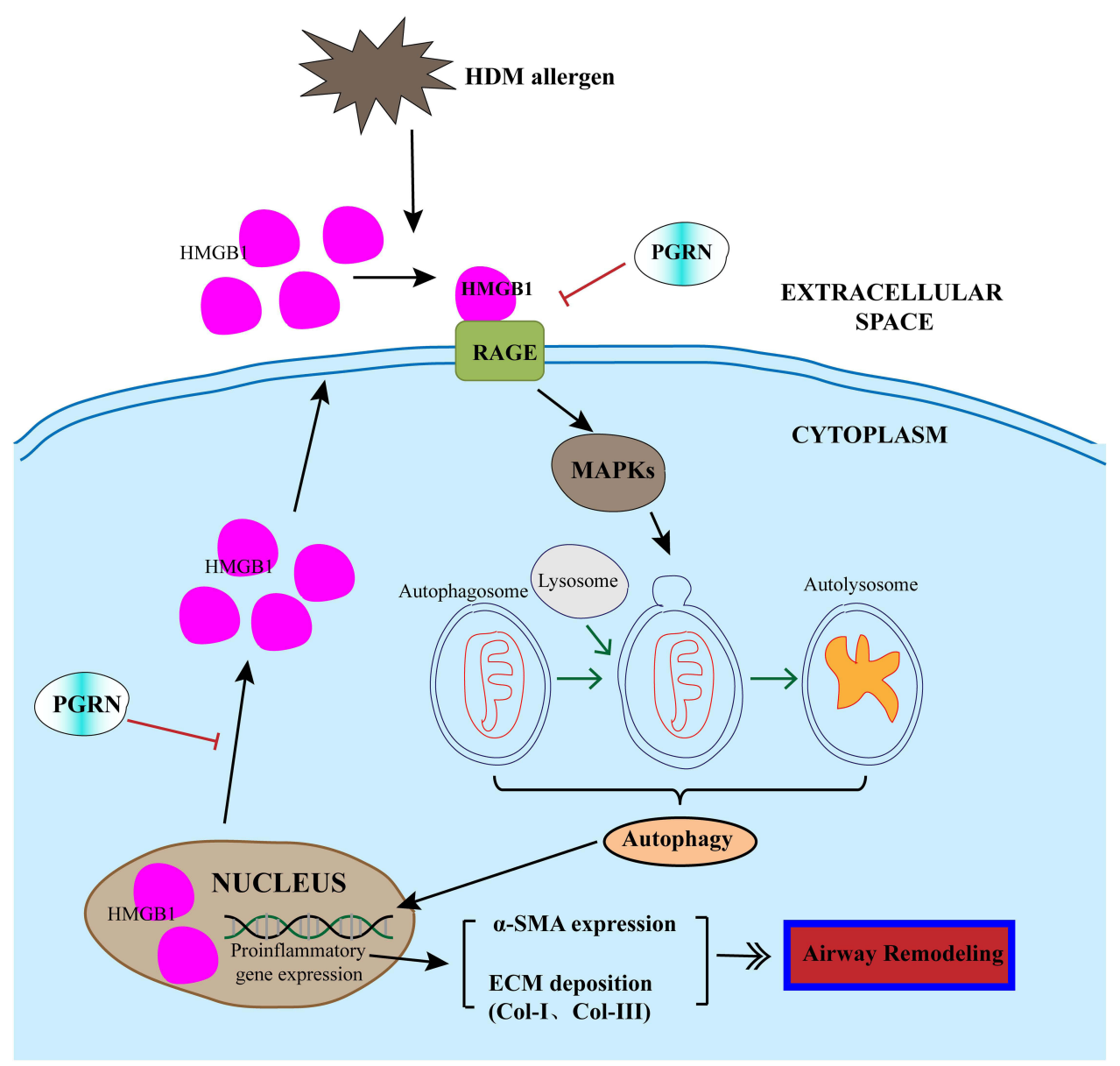

Figure 8 Schematic diagram illustrated the mechanism that HMGBI is involved in the inhibition of PGRN on autophagic activity in HDM-induced airway remodeling. HDM exposure triggered the activation of HMGBI and subsequent RAGE/MAPKs signaling pathway in airway epithelial cells, thus potentiating autophagy process and upregulating the expression of $\alpha$-SMA and collagen proteins, then the formation of airway remodeling. PGRN could overturn HDM-induced HMGBI overexpression, and further restrained autophagy and alleviated ECM production. Black lines: facilitation; Red lines: inhibition.

B). Altogether, the results show that PGRN prevents fibrotic process in airway epithelia through the reduction of autophagy in a manner dependent on HMGB1 suppression, as illustrated in Figure 8.

\section{Discussion}

Airway remodeling, an independent and major pathology that describes the disruption of airway wall tissues, is an important factor for aggravating the progression of asthma. Hitherto, there have not been clinically satisfactory treatments for asthmatic airway remodeling. PGRN is regarded as a therapeutic agent for its anti-inflammatory and antifibrotic abilities in various diseases. ${ }^{27,28}$ But little is known about the action of PGRN in airway remodeling under asthmatic conditions. In this study, we provided the evidence that PGRN attenuated collagen synthesis and fibrotic process in HDM-induced chronic asthma model. Moreover, PGRN could downregulate autophagy via
HMGB1 suppression that was considered to mainly contribute to airway remodeling in asthma. Although PGRN has not yet been applied to asthmatic patients, our study may provide a new theoretical and experimental basis for clinical treatment of asthma.

PGRN, also known as granulin-epithelium precursor (GEP), proelithelin, or prostate cancer cell-derived growth factor (PCDGF), is an autocrine growth factor with multiple biological functions. ${ }^{6}$ Notably, many studies have suggested the defensive properties of PGRN on inflammatory response in asthma. ${ }^{29}$ Intranasal administration of recombinant PGRN successfully reduced airway hyperresponsiveness by inhibiting the degradation of IKB- $\alpha$ in ovalbumin-challenged mice. ${ }^{30}$ Park et al reported that serum PGRN levels were lower in asthmatic subjects compared with those in healthy controls, thus serving as an indicator for the severity of neutrophil inflammation in asthma. ${ }^{31}$ Based on these aforementioned observations, we speculate that decreased endogenous PGRN 
may be a potential inducer for asthma pathogenesis. Therefore, in the present study, we applied exogenous PGRN to the airways of HDM-challenged asthmatic mice. Our results revealed that PGRN treatment significantly suppressed airway inflammation, collagen accumulation, smooth muscle hyperplasia/ hypertrophy, and submucosal gland hyperproduction in asthmatic mice upon HDM irritations, accompanied by the downregulation of fibrosis markers $\alpha$-SMA and collagen-I in mice lungs. These findings suggested the protective ability of PGRN against HDM-induced airway remodeling and fibrosis.

It is known that HDM allergens are a key source of environmental allergens. HDM-derived allergens such as Derp 1 and Derp 2 are considered to be major triggers that activate airway epithelial cells and promote the release of proinflammatory cytokines via their protease activities, which initiate the primary phase during asthmatic process. ${ }^{32}$ For another, damaged epithelial cells exposed to HDM produce a lot of pro-fibrotic mediators such as TGF- $\beta 1$ that modulate the formation of ASM and ECM proteins. TGF- $\beta 1$ renders positive influence on subepithelial fibrosis, EMT induction, and myofibroblast differentiation, which is strongly involved in the changes of airway structures. ${ }^{33}$ Consistently, higher expression of TGF- $\beta 1$ and another mesenchymal marker MMP9 were detected in HDM-challenged mice compared with the controls. In the in vivo experiments of the current study, we found that TGF- $\beta 1$ stimulation drove matrix production and collagen accumulation through upregulating ECM and EMT markers expression in BEAS-2B cells. TGF$\beta 1$-induced EMT in airway epithelial cells is an underlying cause which leads to fibrosis of the airways. Following HDM allergens insult, damaged airway epithelium can be induced into mesenchymal transdifferentiation which promotes disrupted adherens junctions. This inappropriate airway repair further facilitates fibrogenesis involved in airway remodeling. ${ }^{34}$ Additionally, in this study, more serious tendencies of fibrotic process were displayed in airway epithelia with longer-term TGF- $\beta 1$ stimuli, which were yet abrogated by PGRN treatment. Collectively, our results confirm the inhibitory impact of PGRN on TGF- $\beta 1$-mediated fibrosis that essentially contributes to abnormal transformations in airway wall composition in HDM-induced chronic asthma.

Autophagy is a "self-eating" regulatory mechanism that disposals metabolic waste and maintains cellular homeostasis in different physiological conditions. Nevertheless, aberrant autophagy can induce type II programmed cell death and may lead to the occurrence of various diseases. ${ }^{35}$ Emerging evidence verify the role of autophagy in asthma pathogenesis. Poon et al showed that severe asthmatics had more autophagosomes in epithelial cells of bronchial biopsy tissues than healthy subjects. ${ }^{36}$ Elevated expression of autophagyrelated gene 5 (Agt5) was found to greatly result in airway collagen synthesis in asthma patients. ${ }^{18}$ Moreover, autophagy activation is considered as a crucial promoter for extracellular matrix deposition in asthmatic airway remodeling. ${ }^{37}$ In the present study, increased expression of autophagy markers LC3, Beclin-1 and p62 was discovered in asthmatic mice and TGF- $\beta 1$-treated cells, which was also actively correlated with collagen accumulation and fibrotic process in airway epithelia. Intriguingly, PGRN alleviated airway remodeling coupled with the suppression of autophagy both in our in vivo and in vitro experiments, which was also confirmed by an autophagy inhibitor 3-MA treatment. These findings demonstrate that negative regulation of PGRN on autophagy is a possible mechanism of reducing airway remodeling. Actually, accumulating data provided the evidence supporting the function of PGRN as a novel regulator on the modulation of autophagic activity. PGRN deficiency in aged mice led to aggregation of the autophagy markers p62/ubiquitin in neuronal and glial cells via disturbing autophagy-lysosomal pathway. ${ }^{38,39}$ But a research from Zhou et al reported that PGRN prevented podocyte injuries by promoting autophagic activity in diabetic nephropathy. ${ }^{40}$ Therefore, the discrepancy about the role of PGRN on autophagy might depend on diverse types of tissues and cells under specific pathophysiological conditions.

Mechanically, there are many signaling pathways involved in the regulation of autophagy. As a recognized inflammatory mediator, HMGB1 released by injured cells plays critical roles in immunity, inflammation and fibrosis by eliciting autophagy. ${ }^{21,22,25}$ A study by Hou et al showed that elevated HMGB1 activity led to airway remodeling in OVA-challenged asthma model. ${ }^{41}$ Likewise, we herein demonstrated that HMGB1 expression was upregulated following HDM stimuli in mice, whilst silencing HMGB1 inhibited TGF- $\beta 1$-mediated collagen synthesis and fibrosis. Also, increased levels of extracellular HMGB1 improved autophagy flux in airway epithelia under fibrotic conditions, which were concomitant with fibrotic process. These results identified the functions of HMGB1 on HDM-induced airway remodeling and pathological fibrosis through upregulating autophagy. Recently, some researchers sought to link the protective role of PGRN to HMGB1 suppression. In LPS-induced acute kidney injury model, PGRN pretreatment effectively reduced the expression and nucleocytoplasmic translocation of HMGB1 in renal tubular epithelial cells of mice. ${ }^{42}$ Similarly, our data in the present study clarified that HDM-mediated HMGB1 overexpression in airway tissues of asthmatic mice was obviously abolished by PGRN 
treatment. It was thus likely that HMGB1 was a target in PGRN-exerted antagonistic effect against airway remodeling in HDM-induced asthma. We further demonstrated that the improvement of collagen synthesis and autophagic activity by PGRN was offset when extracellular HMGB1 levels were elevated. These findings indicate that the protection of PGRN against asthmatic airway remodeling via downregulating autophagy is undoubtedly attributed to HMGB1 suppression. In addition, our results revealed the reduction of RAGE expression and the inactivation of MAPKs (JNK, ERK1/2, p38) signaling following HMGB1 suppression in PGRN-treated asthmatic mice. Notably, RAGE is a well-accepted receptor bound by HMGB1 to mediate allergic inflammation in asthma. ${ }^{43}$ HMGB1-RAGE axis has also been proved to induce autophagy under diverse disorders. ${ }^{44,45}$ So we speculated that PGRN may render protective ability through inhibiting HMGB1-RAGE and downstream MAPKs signaling during HDM-induced asthma, which was partly in accordance with a previous study by Liu et al. ${ }^{46}$ There were some limitations that we only evaluated the changes of RAGE expression in PGRN-treated mice lungs under HDM exposure. The correlation between PGRN and other HMGB1-mediated signaling pathways is warranted to be elucidated in further study.

\section{Conclusions}

In conclusion, inhaled HDM allergens trigger aberrant autophagy of airway epithelial cells to accelerate airway remodeling. PGRN effectively ameliorates the HDMinduced airway remodeling in terms of collagen production and fibrosis, which is associated with reduction of autophagy by inhibiting HMGB1. Our data provides evidence for PGRN as a promising therapeutic option of HDM-induced chronic asthma.

\section{Acknowledgments}

The authors thank Xuelian Fu and Yongchao Cai for their technical assistance.

\section{Funding}

This work was supported by grants from the National Natural Science Foundation of China (No. 82070076), the Natural Science Foundation of Jiangxi Province (No. 20192ACBL20050) and the Outstanding Clinical Discipline Project of Shanghai Pudong (No. PWYgy2018-06).

\section{Disclosure}

The authors report no conflicts of interest in this work.

\section{References}

1. Mims JW. Asthma: definitions and pathophysiology. Int Forum Allergy Rhinol. 2015;5(Suppl 1):S2-6. doi:10.1002/alr.21609

2. Hough KP, Curtiss ML, Blain TJ, et al. Airway remodeling in asthma Front Med. 2020;7:191. doi:10.3389/fmed.2020.00191

3. Hirota N, Martin JG. Mechanisms of airway remodeling. Chest. 2013;144(3):1026-1032. doi:10.1378/chest.12-3073

4. Halwani R, Al-Muhsen S, Al-Jahdali H, Hamid Q. Role of transforming growth factor-beta in airway remodeling in asthma. Am J Respir Cell Mol Biol. 2011;44(2):127-133. doi:10.1165/rcmb.2010-0027TR

5. Berair R, Brightling CE. Asthma therapy and its effect on airway remodelling. Drugs. 2014;74(12):1345-1369.

6. Daniel R, He Z, Carmichael KP, Halper J, Bateman A. Cellular localization of gene expression for progranulin. $J$ Histochem Cytochem. 2000;48(7):999-1009. doi:10.1177/002215540004800713

7. Xie W, Lu Q, Wang K, et al. miR-34b-5p inhibition attenuates lung inflammation and apoptosis in an LPS-induced acute lung injury mouse model by targeting progranulin. J Cell Physiol. 2018;233 (9):6615-6631. doi:10.1002/jcp.26274

8. Lui H, Zhang J, Makinson SR, et al. Progranulin deficiency promotes circuit-specific synaptic pruning by microglia via complement activation. Cell. 2016;165(4):921-935. doi:10.1016/j. cell.2016.04.001

9. $\mathrm{Wu} \mathrm{H}$, Siegel RM. Medicine. Progranulin resolves inflammation. Science. 2011;332(6028):427-428. doi:10.1126/science.1205992

10. Zhou M, Tang W, Fu Y, et al. Progranulin protects against renal ischemia/reperfusion injury in mice. Kidney Int. 2015;87 (5):918-929. doi:10.1038/ki.2014.403

11. Lee KY, Park SY, Park S, et al. Progranulin protects lung epithelial cells from cigarette smoking-induced apoptosis. Respirology. 2017;22 (6):1140-1148. doi:10.1111/resp.13023

12. Wei J, Zhang L, Ding Y, et al. Progranulin promotes diabetic fracture healing in mice with type 1 diabetes. Ann N Y Acad Sci. 2020;1460 (1):43-56. doi:10.1111/nyas. 14208

13. Yoo W, Lee J, Noh KH, et al. Progranulin attenuates liver fibrosis by downregulating the inflammatory response. Cell Death Dis. 2019;10 (10):758. doi:10.1038/s41419-019-1994-2

14. Sasaki T, Shimazawa M, Kanamori H, et al. Effects of progranulin on the pathological conditions in experimental myocardial infarction model. Sci Rep. 2020;10(1):11842. doi:10.1038/s41598-02068804-7

15. Li SS, Zhang MX, Wang Y, et al. Reduction of PGRN increased fibrosis during skin wound healing in mice. Histol Histopathol. 2019;34(7):765-774.

16. Wang K, Zhang T, Lei Y, et al. Identification of ANXA2 (annexin A2) as a specific bleomycin target to induce pulmonary fibrosis by impeding TFEB-mediated autophagic flux. Autophagy. 2018;14 (2):269-282. doi:10.1080/15548627.2017.1409405

17. Neill T, Schaefer L, Iozzo RV. Instructive roles of extracellular matrix on autophagy. Am J Pathol. 2014;184(8):2146-2153. doi:10.1016/j. ajpath.2014.05.010

18. Poon AH, Choy DF, Chouiali F, et al. Increased autophagy-related 5 gene expression is associated with collagen expression in the airways of refractory asthmatics. Front Immunol. 2017;8:355. doi:10.3389/ fimmu.2017.00355

19. Hammad H, Lambrecht BN. Barrier epithelial cells and the control of type 2 immunity. Immunity. 2015;43(1):29-40. doi:10.1016/j. immuni.2015.07.007

20. Imbalzano E, Quartuccio S, Di Salvo E, Crea T, Casciaro M, Gangemi S. Association between HMGB1 and asthma: a literature review. Clin Mol Allergy. 2017;15(1). doi:10.1186/s12948-017-0068-1

21. Li J, Zeng C, Zheng B, et al. HMGB1-induced autophagy facilitates hepatic stellate cells activation: a new pathway in liver fibrosis. Clin Sci. 2018;132(15):1645-1667. doi:10.1042/CS20180177 
22. Zhang C, Hu J, Wang W, Sun Y, Sun K. HMGB1-induced aberrant autophagy contributes to insulin resistance in granulosa cells in PCOS. FASEB J. 2020;34(7):9563-9574. doi:10.1096/ fj.202000605RR

23. Lee KS, Kim SR, Park HS, et al. A novel thiol compound, $\mathrm{N}$-acetylcysteine amide, attenuates allergic airway disease by regulating activation of NF-kappaB and hypoxia-inducible factor-1alpha. Exp Mol Med. 2007;39:756-768. doi:10.1038/emm.2007.82

24. Kang R, Livesey KM, Zeh HJ, Loze MT, Tang D. HMGB1: a novel Beclin 1-binding protein active in autophagy. Autophagy. 2010;6 (8):1209-1211. doi:10.4161/auto.6.8.13651

25. Chai W, Ye F, Zeng L, Li Y, Yang L. HMGB1-mediated autophagy regulates sodium/iodide symporter protein degradation in thyroid cancer cells. J Exp Clin Cancer Res. 2019;38(1):325. doi:10.1186/ s13046-019-1328-3

26. Shim EJ, Chun E, Lee HS, et al. The role of high-mobility group box-1 (HMGB1) in the pathogenesis of asthma. Clin Exp Allergy. 2012;42(6):958-965. doi:10.1111/j.1365-2222.2012.03998.x

27. Li X, Cheng S, Hu H, et al. Progranulin protects against cerebral ischemia-reperfusion (I/R) injury by inhibiting necroptosis and oxidative stress. Biochem Biophys Res Commun. 2020;521(3):569-576. doi:10.1016/j.bbrc.2019.09.111

28. Zhou D, Zhou M, Wang Z, et al. PGRN acts as a novel regulator of mitochondrial homeostasis by facilitating mitophagy and mitochondrial biogenesis to prevent podocyte injury in diabetic nephropathy. Cell Death Dis. 2019;10(7):524. doi:10.1038/s41419-019-1754-3

29. Pogonowska M, Poniatowski LA, Wawrzyniak A, Krolikowska K, Kalicki B. The role of progranulin (PGRN) in the modulation of anti-inflammatory response in asthma. Central-Eur J Immunol. 2019;44(1):97-101. doi:10.5114/ceji.2019.83267

30. Chiba Y, Danno S, Suto R, et al. Intranasal administration of recombinant progranulin inhibits bronchial smooth muscle hyperresponsiveness in mouse allergic asthma. Am J Physiol Lung Cell Mol Physiol. 2018;314(1):L215-L223. doi:10.1152/ajplung.00575.2016

31. Park SY, Hong GH, Park S, et al. Serum progranulin as an indicator of neutrophilic airway inflammation and asthma severity. Ann Allergy, Asthma Immunol. 2016;117(6):646-650. doi:10.1016/j. anai.2016.09.437

32. Zhang J, Chen J, Robinson C. Cellular and molecular events in the airway epithelium defining the interaction between house dust mite group 1 allergens and innate defences. Int J Mol Sci. 2018;19 (11):3549. doi:10.3390/ijms19113549

33. Royce SG, Cheng V, Samuel CS, Tang ML. The regulation of fibrosis in airway remodeling in asthma. Mol Cell Endocrinol. 2012;351 (2):167-175. doi:10.1016/j.mce.2012.01.007

34. Rout-Pitt N, Farrow N, Parsons D, Donnelley M. Epithelial mesenchymal transition (EMT): a universal process in lung diseases with implications for cystic fibrosis pathophysiology. Respir Res. 2018;19(1):136. doi:10.1186/s12931-018-0834-8
35. Mazure NM, Pouyssegur J. Hypoxia-induced autophagy: cell death or cell survival? Curr Opin Cell Biol. 2010;22(2):177-180. doi:10.1016/ j.ceb.2009.11.015

36. Poon AH, Chouiali F, Tse SM, et al. Genetic and histologic evidence for autophagy in asthma pathogenesis. J Allergy Clin Immunol. 2012;129(2):569-571. doi:10.1016/j.jaci.2011.09.035

37. McAlinden KD, Deshpande DA, Saeid Ghavami DX, et al. Autophagy activation in asthma airways remodeling. Am $J$ Respir Cell Mol Biol. 2019;60(5):541-553. doi:10.1165/rcmb.2018-0169OC

38. Tanaka Y, Chambers JK, Matsuwaki T, Yamanouchi K, Nishihara M. Possible involvement of lysosomal dysfunction in pathological changes of the brain in aged progranulin-deficient mice. Acta Neuropathologica Commun. 2014;2:78. doi:10.1186/s40478-0140078-x

39. Gotzl JK, Colombo AV, Fellerer K, et al. Early lysosomal maturation deficits in microglia triggers enhanced lysosomal activity in other brain cells of progranulin knockout mice. Mol Neurodegener. 2018;13(1):48. doi:10.1186/s13024-018-0281-5

40. Zhou D, Zhou M, Wang Z, et al. Progranulin alleviates podocyte injury via regulating CAMKK/AMPK-mediated autophagy under diabetic conditions. J Mol Med (Berl). 2019;97(11):1507-1520. doi:10.1007/s00109-019-01828-3

41. Hou C, Kong J, Liang Y, et al. HMGB1 contributes to allergen-induced airway remodeling in a murine model of chronic asthma by modulating airway inflammation and activating lung fibroblasts. Cell Mol Immunol. 2014;12(4):409-423. doi:10.1038/ cmi.2014.60

42. Xu X, Gou L, Zhou M, et al. Progranulin protects against endotoxin-induced acute kidney injury by downregulating renal cell death and inflammatory responses in mice. Int Immunopharmacol. 2016;38:409-419. doi:10.1016/j.intimp.2016.06.022

43. Perkins TN, Donnell ML, Oury TD. The axis of the receptor for advanced glycation endproducts in asthma and allergic airway disease. Allergy. 2020;76:1350.

44. Mou K, Liu W, Han D, Li P. HMGB1/RAGE axis promotes autophagy and protects keratinocytes from ultraviolet radiation-induced cell death. J Dermatol Sci. 2017;85(3):162-169. doi:10.1016/j. jdermsci.2016.12.011

45. Wu CZ, Zheng JJ, Bai YH, Xia P, Zhang HC, Guo Y. HMGB1/ RAGE axis mediates the apoptosis, invasion, autophagy, and angiogenesis of the renal cell carcinoma. Onco Targets Ther. 2018;11:4501-4510. doi:10.2147/OTT.S167197

46. Liu L, Guo H, Song A, et al. Progranulin inhibits LPS-induced macrophage M1 polarization via NF-кB and MAPK pathways. BMC Immunol. 2020;21(1):32. doi:10.1186/s12865-020-00355-y
Journal of Inflammation Research

\section{Publish your work in this journal}

The Journal of Inflammation Research is an international, peerreviewed open-access journal that welcomes laboratory and clinical findings on the molecular basis, cell biology and pharmacology of inflammation including original research, reviews, symposium reports, hypothesis formation and commentaries on: acute/chronic inflammation; mediators of inflammation; cellular processes; molecular mechanisms; pharmacology and novel anti-inflammatory drugs; clinical conditions involving inflammation. The manuscript management system is completely online and includes a very quick and fair peerreview system. Visit http://www.dovepress.com/testimonials.php to read real quotes from published authors. 